http://jmscr.igmpublication.org/home/ ISSN (e)-2347-176x ISSN (p) 2455-0450 crossref DOI: https://dx.doi.org/10.18535/jmscr/v8i5.07

\author{
Journal Of Medical Science And Clinical Research

\title{
Conservative Treatment of Unilateral Condylar Fracture in a Pediatric Patient
}

\author{
Authors \\ Bibina C P $\mathbf{P}^{1}$, Rita Zarina. $\mathrm{A}^{2}$, Suchithra $\mathrm{M} \mathrm{S}^{3}$, Anjani S Mallya ${ }^{4}$ \\ ${ }^{1}$ Junior resident, Dept. of Pediatric and Preventive Dentistry, Government Dental College, Thiruvananthapuram, \\ Kerala, India \\ ${ }^{2}$ Professor and Head, Dept. of Pediatric and Preventive Dentistry, Government Dental College, Thiruvananthapuram, \\ Kerala, India \\ ${ }^{3}$ Additional Professor, Dept. of Pediatric and Preventive Dentistry, Government Dental College, Thiruvananthapuram, \\ Kerala, India \\ ${ }^{4}$ Junior Resident, Dept. of Pediatric and Preventive Dentistry, Government Dental College, Thiruvananthapuram, \\ Kerala, India
}

\begin{abstract}
The prevalence of $C F$ in children is low and treatment must focus on possible long-term effects on the growing facial skeleton and soft tissues. Conservative treatment, including close observation, soft diet, physiotherapy, and functional appliances, are advised in most cases. The immense capacity for healing in children within the shortest possible time showing minimum complications, and the inherent ability to adapt to new situations are quite different from what we seen in adults.

This article aims to review the preferable options for the management of mandibular condylar fracture in children and to discuss a case report where satisfactory remodelling of condyle occurred and normal occlusion and jaw movements were obtained.
\end{abstract}

Key words: Condylar fracture; Conservative treatment; TMJ ankylosis; Condylar remodelling.

\section{Introduction}

Facial injuries in children are much less common than adults. Mandibular fractures are the most common facial skeletal injury in pediatric patients ${ }^{[1]}$. Of mandibular fracture, condyle is the most common site of fracture in pediatric maxillofacial trauma (More than 50\%), followed by parasymphysis, body and angle ${ }^{[2]}$. The prevalence of $\mathrm{CF}$ in children is low and treatment must focus on possible long-term effects on the growing facial skeleton and soft tissues.

Compared with their adult counterparts, pediatric condylar fractures are more commonly managed conservatively $^{[3]}$.

\section{Classification}

Lindhal classification 1977

Anatomic location of the fracture

- Condylar head

- Condylar neck

- Subcondylar

Relationship of condylar fragment to mandible

- Nondisplaced

- Deviated

- Displacement with medial or lateral overlap

- Displacement with anterior or posterior overlap

- No contact between fractured segments

Relationship of condylar head \& fossa

- Nondisplaced 
- Displacement

- Dislocation

\section{Clinical signs and symptoms (Table 1)}

Both TMJs must be assessed with regard to pain, clicking, locking and crepitus. Maximum mouth opening and deviations from the midline during opening should be recorded and excursions in the horizontal plane must be measured ${ }^{[4]}$.

\section{Diagnostic aids}

At first consultation, the signs and symptoms must be assessed and an orthopantomogram, together with a modified anterior posterior projection according to Towne, can be taken. The site and level of the fracture can be accessed from this.

Post-traumatic radiographic tracings of the condylar head and neck can be taken after 6 weeks, six months time interval. By super positioning the tracings at the dorsal side and angle area, the differences in length of the ascending ramus can be measured. On orthopantomograms, antero-posterior and oblique transcranial views, small fragments cannot always be detected and a CT scan certainly will give better information ${ }^{[5]}$.

\section{Adult v/s children}

The mandible comparatively undersized and protected by the larger cranium in early childhood ${ }^{[6]}$. Clinical signs of shock may occur due to small blood volume. Because of small size of airway laryngeal edema or retro position of base of tongue may produce sudden obstruction.

They have immense capacity for healing in children within the shortest possible time with minimum of complications, the assistance that growth can give, and the inherent ability to adapt to new situation are quite different from what we see in adults ${ }^{[1]}$. Young bone possesses unique physical properties and bone fragments in children may become partially united as early as 4 days and fractures become difficult to reduce by seventh day ${ }^{[7]}$. Non-union or fibrous union rarely occurs in children and excellent remodeling occurs under the influence of masticatory stresses even when there is imperfect apposition of bone surfaces ${ }^{[8]}$.Between 2-4 years sufficient number of fully formed deciduous teeth is present facilitating application of arch bars or eyelet wires.

While doing open reduction and fixation presence of tooth buds throughout the body of mandible must be a consideration as trauma to developing tooth buds may result in failure of eruption of permanent teeth and hence narrow alveolar ridge ${ }^{[9]}$. In a more recent paper Blackwood (1961) has stated that it is likely that the vascular channels in the mandibular condyle disappear at the age of 2 years and that after this age there is much less likelihood of permanent damage to mandibular growth following injury to the condylar process. The prognosis appears to be good provided the fractured fragments are in contact, the degreeof contact being directly related to the end result ${ }^{[10]}$.

\section{Remodelling}

In many studies concerned with condyle fractures in children, the phenomena of condylar regeneration and adaptive remodeling have been ascribed to processes occurring in the articular and proliferative zone of the condyle. Bone fragments in children may become partially united as early as 4 days and fractures become difficult to reduce by seventh day. This results in need for different forms of fixation as early as possible for comparatively shorter duration of time.

Non-union or fibrous union rarely occurs in children and excellent remodeling occurs under the influence of masticatory stresses even when there is imperfect apposition of bony surfaces ${ }^{[7]}$. The cranial fragment undergoes resorption and the caudal fragment progressively regenerates, although the condylar remodeling to the original morphology can only be expected in children, not in adolescents or adults. Especially in situations in which the cranial fragment is lost in a growing patient, a complete recovery of oral functions is mandatory to ensure a further normal growth of the mandible. The therapy must be carefully done as severe long-term complications can occur. The most dangerous complication is real ankylosis of the Temporomandibular joint (TMJ) ${ }^{[11]}$. 
Table 1

\begin{tabular}{|c|c|}
\hline Unilateral condylar fracture & Bilateral condylar fracture \\
\hline $\begin{array}{l}\text { - Facial asymmetry of the involved side - posterior and } \\
\text { lateral shift of mandible towards the affected side and } \\
\text { dental midline deviation toward the ipsilateral side. } \\
\text { Premature occlusion in molar region - upward pull of the } \\
\text { mandible by elevator } \\
\text { Localized pain or swelling of the TMJ and cross bite on } \\
\text { the affected side } \\
\text { Shift of occlusion towards the ipsilateral side with } \\
\text { possible cross bite and lack of condylar movements upon } \\
\text { palpation. } \\
\text { Difficulty in excursion and protrusion }\end{array}$ & $\begin{array}{l}\text { - } \quad \text { Retrognathism } \\
\text { Anterior open bite - upward pull of the mandible by } \\
\text { lateral pterygoid muscle }\end{array}$ \\
\hline
\end{tabular}

\section{Management}

The two main goals for treatment of condylar fracture in pediatric patients are,

(1) Preservation of function

(2) Maintenance of ramus height ${ }^{[9]}$.

When this is achieved normal growth usually occurs. For the treatment of mandibular condyle fractures in children, both non-surgical and surgical approaches have been recommended, and the appropriate mode of therapy still remains a point of controversy. The location and degree of displacement of condylar fractures in primary and mixed dentition stage is not a very useful variable for developing treatment plan (Table 2). Rather the amount of interincisal opening, dental age, occlusion and level of pain must be assessed carefully. If these are normal, close observation and blenderized diet can be the treatment option ${ }^{[9]}$.

\section{Table 2}

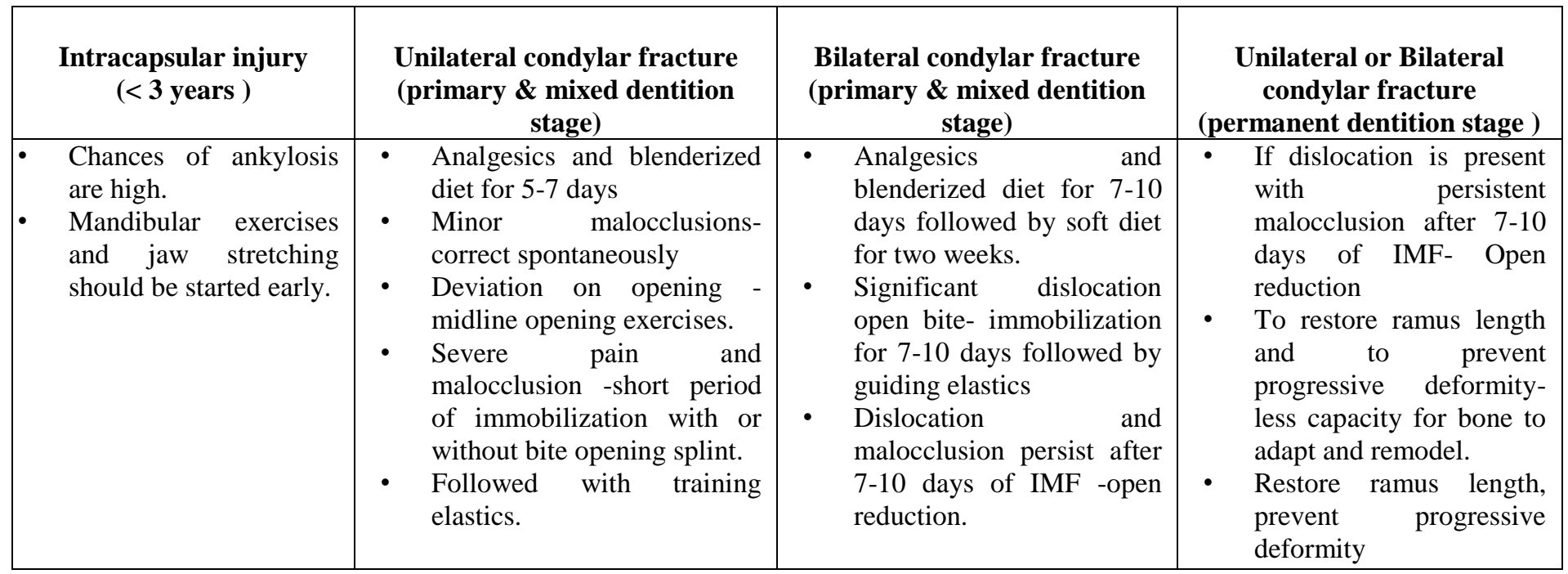

Functional treatment, maxillomandibular fixation and open reduction are the three modes of treatment that have been suggested in the literature ${ }^{[12]}$. Conservative treatment, including close observation, soft diet, physiotherapy, maxillomandibular fixation, training elastics, bite opening splints and functional appliances, are advised in most cases, while others prefer open reduction with internal fixation. Restoration of normal symmetric jaw function provides best chance for normal growth. In children the final result is determined not merely by initial treatment but by the effect that growth has on form and function ${ }^{[13]}$.

\section{Newer trends in treatment}

With the advent of better investigative facilities like CT scan, 3D reconstruction, and newer airway management techniques with reliable anesthesia techniques and specifically introduction of mini and microplates ORIF of pediatric facial fractures is 
getting commoner. Also, resorbable materials have been made available as a fixation option for pediatric craniomaxillofacial fracture management. According to Peterson with the exception of mandibular condyle fractures judicious use of ORIF is preferable to the closed reduction and immobilization techniques with splints when treating fractures in the deciduous and mixed dentition $^{[14]}$.

\section{Sequelae}

Injuries involving the TMJ can result in growth disturbance, facial asymmetry, malocclusion, limited mouth opening, chronic pain, and loss of ramus height. The most dangerous complication is real ankylosis of the fractures may give rise to serious problems, for example growth disturbances of the face on the injured side and TMJ disorders on the injured as well as the non-injured side. Growth abnormalities may occur as result of fracture dislocation of condyle due to elimination of 'functional matrix' of lateral pterygoid function, trismus or ankylosis ${ }^{[9]}$.

In monolateral fractures, the risk consists in a unilateral reduction in mandibular growth, which in an advanced age may require complex orthodontic or surgical procedures. In bilateral fractures, a severe class II may occur due to mandibular defect, leading to both functional and esthetic discomfort. These potential complications make identification, appropriate treatment, and long-term follow-up of condylar fractures in the developing mandible essential. Both cosmetic and functional sequelae may occur ${ }^{[13]}$.

In Lund's study, mandibular growth was generally greater on fractured side than non-fractured side so that the fractured ramus which was initially shorter had greater incremental growth rate, so that possible disproportion between two sides reduced with time. This was evident when the distance between chin point to condyle was measured ${ }^{[15]}$.

\section{Case Report}

6 yrs old female patient was brought by her father to our department with an alleged history of fall, two days back from a wooden cot at home. She presented with pain on chin region and restricted mouth opening. She had consulted a nearby general hospital and was referred to dental casuality, Government Medical College, Trivandrum. No history of loss of consciousness, seizures, head ache or ENT bleeding was reported. The patient was advised to do ice compression on chin and no other active interventions were done at the casuality. For further management, the patient was referred to our institute.

On extra oral examination, there was a hematoma on the chin .Swelling and tenderness over the left side of the face were also noted. On palpation, tenderness and mild depression was felt over the left pre auricular region. The prominence of the condyle was not felt on the left TMJ region.

Maxilla and mandible appeared to be normal. Occlusion was stable but mouth opening was restricted to $25 \mathrm{~mm}$ (Figure1).

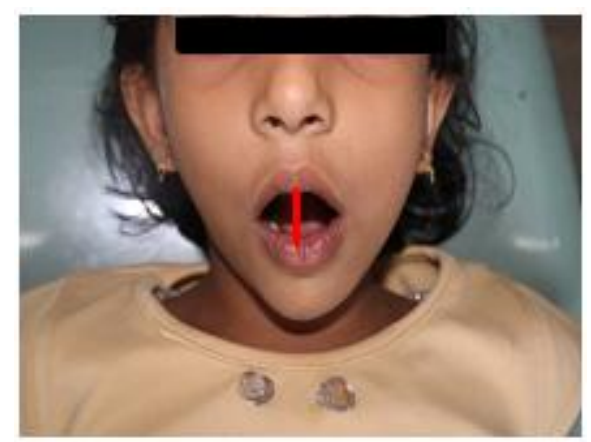

Figure 1 Mouth opening $25 \mathrm{~mm}$

On intra oral examination, pulpally involved 54, 55, $64,74,75,85$ and multiple teeth with dentinal caries were also noted. Angle's Class I malocclusion with lower anterior crowding and tongue thrusting habit were present.

Panoramic radiograph (Figure 2) revealed a left subcondylar fracture with medial displacement. According to Lindhal classification, based on anatomic location of the fracture this type of injury come under subcondylar fracture. The vertical dimension and occlusion were preserved. 


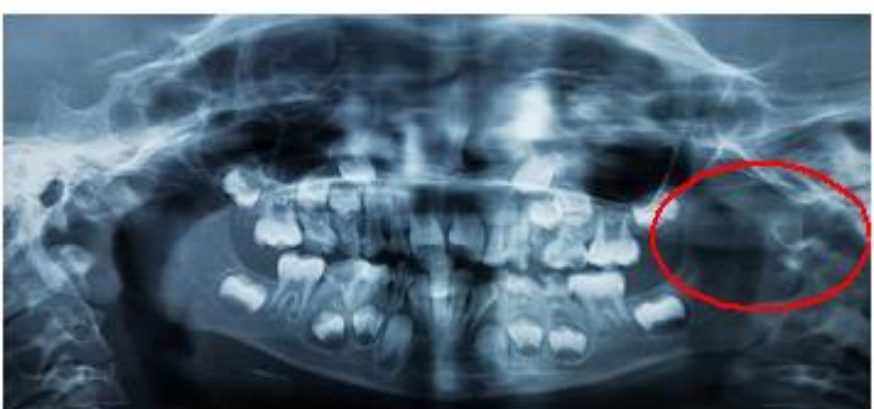

Figure 2 OPG showing left subcondylar fracture with medial displacement

Considering immense capacity for healing in children, the unique physical properties in young bones, assistance that growing children can give, the possible trauma to the developing tooth buds and above all a consultation from the orthodontist and maxillofacial surgeon lead us to avoid the ORIF approach, in favour of closed reduction.

The treatment primarily consisted of reassurance and explanation to both the parent and the child. Parents were advised to watch their child carefully, and make sure that she closed the mouth in maximum occlusion. Series of functional exercises were suggested for 15 minutes, four times a day. The prescribed functional exercises were maximum mouth opening, maximum protrusive movement, and maximum right and left lateral movements. We also advised to use chewing gum only under strict parental supervision. Instructions were given to maintain good oral hygiene and an antiinflammatory analgesic was also prescribed.

Patient was recalled after three days. She showed a good 3 finger width mouth opening (Figure 3) which was improved from 25 to $40 \mathrm{~mm}$ and with reduced tenderness. No other abnormalities or midline deviation were noted. Full mouth oral prophylaxis was done and all the carious teeth were restored. Teeth with clinically and radiographically poor prognosis were extracted and space management was considered. For other pulpally exposed teeth with good prognosis, pulptherapy followed by stainless steel crown was given.

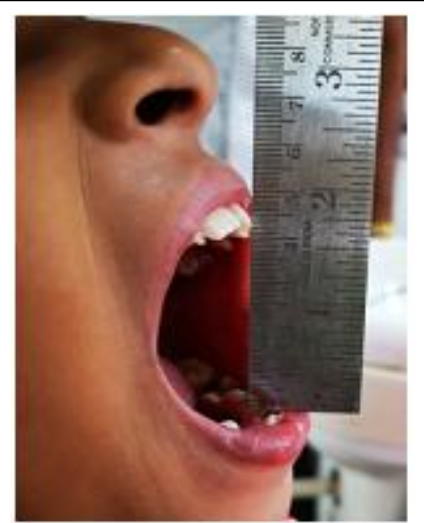

Fig: 3 After three days follow up showed mouth opening about $40 \mathrm{~mm}$

On subsequent reviews patient improved in terms of mouth opening which measures to $40 \mathrm{~mm}$.

The OPG taken 3 months after the trauma did not show any visible fracture line instead gave a strong evidence of a perfectly remodelled fractured segment (Figure 4). The patient was advised to continue functional exercises for a further period of six months.

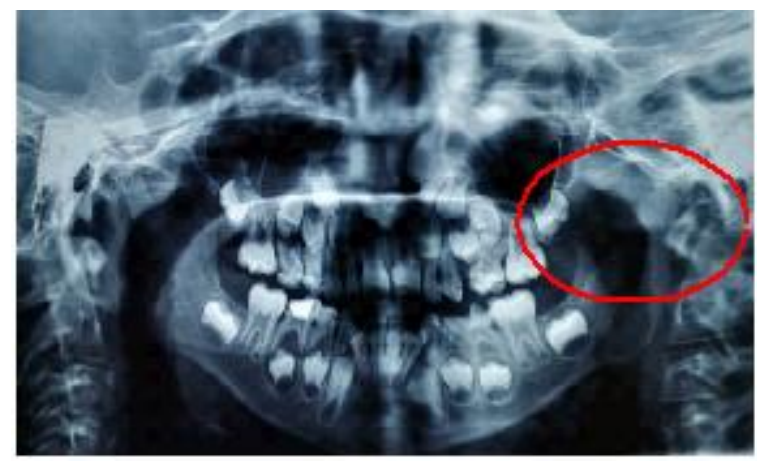

Figure 4 OPG showing perfect left condylar remodelling revealed after 3 months period

\section{Conclusion}

Mandibular fractures in children most commonly occur in the condylar region, followed by parasymphysis and angle. These fractures tend to be minimally displaced and in majority of cases can be treated conservatively. Significantly displaced mandibular fractures are reduced and immobilized using rigid internal fixation, similar to principles used in adults. Fractures in condylar region usually are treated using non-operative therapies as in most cases fracture heals and the condyle is remodelled with successful anatomic and functional results. 


\section{Reference}

1. Kumar D, Trehan M, Vashistha A, Chugh A, Nigam AG, Trehan $\mathrm{M}$, et al. Pediatric Mandibular Fractures: A Review. International Journal of Clinical Pediatric Dentistry. 2009 Aug;2(2):1-5.

2. Posnick JC, Goldstein JA. Surgical Management of Temporomandibular Joint Ankylosis in the Pediatric Population: Plastic and Reconstructive Surgery. 1993 Apr;91(Supplement):791-8.

3. Cassi D, Magnifico M, Di Blasio C, Gandolfini M, Di Blasio A. Functional Treatment of a Child with Extracapsular Mandibular Fracture. Case Reports in Dentistry. 2017;2017:1-5.

4. Hovinga J, Boering G, Stegenga B. Longterm results of nonsurg cal management of condylar fractures in children. :12.

5. (PDF) Long-Term Outcomes After Treatment of Condylar Fracture by Intraoral Access: A Functional and Radiologic Assessment [Internet]. [

6. Goth S, Sawatari Y, Peleg M. Management of pediatric mandible fractures. J Craniofac Surg. 2012; 23:47-56.

7. Kaban LB, Mulliken JB, Murray JE. Facial fractures in children: An analysis of 122 fractures in 109 patients. Plast Reconstr Surg 1977 Jan;59(1):15-20.

8. Güven O, Keskin A. Remodelling following condylar fractures in children. Journal of cranio-maxillo-facial surgery: official publication of the European Association for Cranio-Maxillo-Facial Surgery. 2001 Sep 1;29:232-7.

9. Sharma S, Vashistha A, Chugh A, Kumar D, Bihani U, Trehan M, et al. Pediatric Mandibular Fractures: A Review. Int J Clin Pediatr Dent. 2009;2(2):1-5.

10. BLACKWOOD, J. H. (1961). Temporomandibular Joint Symposium, R.C.S., London.

11. Di Blasio C., Di Blasio A., Pedrazzi G., Anghinoni M., Sesenna E. How does the mandible grow after early high condylectomy? The Journal of Craniofacial Surgery. 2015;26(3):764-771. doi: 10.1097/scs.0000000000001515. [PubMed] [CrossRef] [Google Scholar].

12. Bayat M, Parvin M, Meybodi AA. Mandibular Subcondylar Fractures: A Review on Treatment Strategies. Electron Physician. 2016 Oct 25;8(10):3144-9.

13. Medina A. Functional Appliance Treatment for Bilateral Condylar Fracture in a Pediatric Patient. Pediatric dentistry. 2009 Sep 1;31:432-7.

14. Thoren $H$, lizuka $T$, Hallikainen $D$, Nurminen M, Lindqvist C. An epidemiological study of patterns of condylar fractures in children. Br $\mathrm{J}$ Oral Maxillofac Surg. 1997 Oct;35(5):306-311.

15. Lund K. Mandibular growth and remodeling processes after condylar fracture: A longitudinal roentgencephalometric study. Acta odontol Scand Suppl. 1974;32(64):3117. 